Archived version from NCDOCKS Institutional Repository http://libres.uncg.edu/ir/asu/

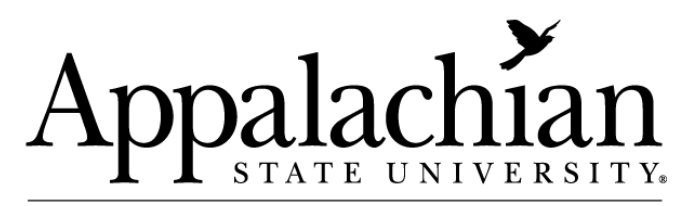

B O O N E, NORT H C A R O L I A

\title{
CALM Gatekeeper Training Is Associated With Increased Confidence In Utilizing Means Reduction Approaches To Suicide Prevention Among College Resident Assistants
}

\author{
By: Melanie R. Rosen, Kurt D. Michael, PhD, and J.P. Jameson, PhD
}

\begin{abstract}
Background: Most suicide prevention programs focus on increasing knowledge regarding the problem of suicide, yet many fail to include information on the science and application of means reduction approaches. In an attempt to address this gap in practice, the Counseling on Access to Lethal Means (CALM) program was developed to educate clinicians on the importance of means reduction interventions. Methods: In the current study, a gatekeeper CALM training was delivered to 167 resident assistants. Confidence levels regarding suicide prevention and means reduction skills were assessed at baseline, post-training, and after a 6-week follow-up. Results: Results were suggestive of medium to large training effects. Though there was a small decay of training effects at follow-up, the effects were durable when compared to baseline levels. Conclusion: Given these findings, future gatekeeper trainings should be provided more consistently to help sustain the effects and data on the implementation of CALM principles should be measured during follow-up assessments.
\end{abstract}

Melanie R. Rosen, Kurt D. Michael \& J. P. Jameson (2020): CALM gatekeeper training is associated with increased confidence in utilizing means reduction approaches to suicide prevention among college resident assistants, Journal of American College Health, DOI:1 0.1080/07448481.2020.1756825. Publisher version of record available at: https://www.tandfonline.com/doi/full/10.1080/07448481.2020.1756825 


\title{
CALM gatekeeper training is associated with increased confidence in utilizing means reduction approaches to suicide prevention among college resident assistants
}

\author{
Melanie R. Rosen, $\mathrm{MA}^{\mathrm{a}, \mathrm{b}}$, Kurt D. Michael, $\mathrm{PhD}^{\mathrm{b}}$, and J. P. Jameson, $\mathrm{PhD}^{\mathrm{b}}$ \\ ${ }^{a}$ Department of Counseling and Clinical Psychology, Teachers College, Columbia University, New York, New York, USA; Department of \\ Psychology, Appalachian State University, Boone, North Carolina, USA
}

\begin{abstract}
Background: Most suicide prevention programs focus on increasing knowledge regarding the problem of suicide, yet many fail to include information on the science and application of means reduction approaches. In an attempt to address this gap in practice, the Counseling on Access to Lethal Means (CALM) program was developed to educate clinicians on the importance of means reduction interventions.

Methods: In the current study, a gatekeeper CALM training was delivered to 167 resident assistants. Confidence levels regarding suicide prevention and means reduction skills were assessed at baseline, post-training, and after a 6-week follow-up.

Results: Results were suggestive of medium to large training effects. Though there was a small decay of training effects at follow-up, the effects were durable when compared to baseline levels. Conclusion: Given these findings, future gatekeeper trainings should be provided more consistently to help sustain the effects and data on the implementation of CALM principles should be measured during follow-up assessments.
\end{abstract}

\section{KEYWORDS}

CALM; gatekeeper training; means reduction; suicide; suicide prevention
Suicide is a vexing public health concern, accounting for 47,173 deaths and ranking as the tenth leading cause of mortality for Americans in 2017. ${ }^{1}$ Since then, suicide rates continue to rise, accounting for approximately 129 deaths per day. ${ }^{2}$ While suicide might never be completely eradicated, innovative suicide prevention programs can likely play a role in stemming this worsening public health crisis. Though the problem of suicide impacts all ages and demographics, colleges and universities are notably impacted by suicide. According to a recent study, the prevalence of suicidal ideation among college students has been consistently on the rise from $5.8 \%$ in 2007 to $10.8 \%$ in $2016-17 .^{3}$ Moreover, a study conducted in 2018 reported that as many as one in four college students had experienced some form of suicidal thought or behavior in the past year. ${ }^{4}$ Therefore, it is imperative that suicide prevention efforts focus on this at-risk population.

\section{Suicide in colleges and universities}

Suicidal thoughts and behaviors tend to affect college students more than other populations for a multitude of reasons; most common of which include feelings of thwarted belongingness and perceived burdensomeness, "coming out" as a member of the queer community, substance use, maladaptive interpersonal behavior and social cognition, and intense academic pressure. $^{5-8}$ Importantly, the transition into college and young adulthood tends to be a period of great change for most adolescents and can lead to significant stress. It has been widely hypothesized that there is a significant link between stress and suicidal ideation, such that an increase in stress is likely to lead to greater suicidal ideation among depressed individuals. ${ }^{9}$

Due to the high prevalence of suicidality among this atrisk population, significant prevention efforts have been implemented by universities nation-wide. ${ }^{10,11}$ In addition to briefing university counseling centers on the rise of suicidality among college students, college resident assistants (RAs) are typically required to undergo suicide prevention training as part of their orientation. Commonly, RAs are informed not to take matters into their own hands, but rather to involve the local police or university counseling center when a student endorses suicidality; this is to protect both the individual in crisis and the RA. However, RAs are trained to be on the lookout for signs that may indicate that an individual is at risk for making a suicide attempt and are given an emergency protocol to follow during these events. ${ }^{10,11}$ The role of these gatekeepers is extremely important in preventing suicide among college students and thus identifying the efficacy of their training is imperative.

Various studies have reported on the effectiveness of RA suicide prevention and intervention training and have found that in general, these trainings are successful at increasing confidence and feelings of preparedness to intervene in a crisis. $^{11,12}$ While these trainings are typically effective at 
preparing RAs to intervene when a resident is suicidal, the type of intervention trainings that are administered tend to be limited. ${ }^{12}$ Many existing suicide prevention programs (e.g., Question, Persuade, Refer [QPR]) emphasize the importance of improving knowledge, attitudes, and perceptions regarding suicide. ${ }^{13}$ Other programs focus on identifying observable risk factors, warning signs, and behaviors in order to quantify an individual's level of risk to help determine appropriate treatment. Common risk factors of suicide generally include a history of previous suicide attempts or non-suicidal self-injury, alcohol or substance abuse, a history of individual or parental psychopathology, low socio-economic status, residing in rural areas, limited social support, and demographic factors such as age, race and gender. ${ }^{14-16}$

While these popular prevention programs illustrate the basics of suicide prevention, many do not account for risk factors such as impulsivity, method lethality, and access to lethal means. As of recently, most RA suicide prevention training programs focused their training on QPR-like interventions. ${ }^{17}$ Due to the high lethality of certain methods and the impulsivity associated with adolescent suicide attempters, the present study sought to identify the effectiveness of a means reduction-based approach to suicide prevention training among a sample of RAs.

\section{Impulsive responding and method lethality}

Impulsive behavior is a major concern among those at risk for suicide. In a study of 82 patients admitted to a psychiatric hospital following a suicide attempt, each was interviewed with the purpose of gaining detailed information about the process leading up to the attempt. Of the 82 participants, nearly $50 \%$ reported that only ten minutes had passed before the initial consideration (thought) to carrying out that particular attempt (action). ${ }^{18}$ Furthermore, in a sample of those who had made an almost-lethal suicide attempt, one-fourth reported spending less than five minutes between the first thought of attempting and the actual attempt. ${ }^{19,20}$ An additional study conducted in 2016 reported that in a sample of 319 suicide attempters, the median duration of the suicidal process was approximately 30 minutes, thus suggesting high levels of impulsivity in suicide attempters. ${ }^{21}$

Extant research suggests a correlation between certain personal characteristics and likelihood of an impulsive suicide attempt. Data suggest that males are more likely than females to attempt suicide impulsively and individuals with severe depression are less likely to attempt suicide impulsively. ${ }^{19}$ These data parallel results from Deisenhammer et al and Kattimani et al, ${ }^{18,21}$ which found that individuals who reported taking longer than ten minutes to make a suicide attempt showed significantly higher intent to die by suicide. These findings are important because they signify that individuals who attempt suicide impulsively may not really want to die, but rather get overwhelmed by their emotions and seek an immediate escape from pain.

Another shortcoming of many prevention programs is the failure to account for method lethality. Of the nearly 129 deaths by suicide that occur daily, approximately half are due to the use of firearms. ${ }^{22,23}$ The rate of handgun deaths determined to be suicides are especially high in certain regions (e.g., $78 \%$ in rural northwest North Carolina). ${ }^{24}$ Furthermore, according to Anestis and Capron, ${ }^{22}$ there are over 300 million privately owned firearms in the United States, which is a number only slightly less than the reported U.S. population of 312.8 million in $2012 .{ }^{25}$ Importantly, more than three-fourths of suicide attempts with a firearm are gunshot wounds to the head, and $76.6 \%$ of all attempts with a firearm are fatal. ${ }^{20}$ Moreover, several studies have shown a $20 \%$ increase in suicide death by firearm among youth ages 15 to 24 years old (2218 deaths in 2012 to 2683 deaths in 2016). ${ }^{23}$

A recent study measuring the relationship between household firearm ownership rates and suicide mortality rates suggest that poorly restricted access to lethal means such as firearms and opioid medications is associated with higher levels of completed suicides, regardless of underlying suicidal ideation. ${ }^{15,20}$ Therefore, ready access to firearms or potentially lethal medications increases the rate at which it is likely to die by suicide. This poses particular challenges for suicide prevention in the United States given the accessibility of lethal means, especially firearms.

Data also suggest a correlation between use of a highly lethal method and the likelihood of making an impulsive attempt. $^{19,26}$ Therefore, the risk of death significantly increases when suicidal individuals show behavioral features of impulsivity and also have access to lethal means. Even when accounting for non-impulsive individuals (such as those with a suicide plan), those who owned a firearm were significantly more likely to have a plan that involved using the firearm than those who did not. ${ }^{27}$ These findings reveal the importance of accounting for impulsivity and access to lethal means when measuring patients' risk levels.

\section{Implementation of means reduction programs}

In an attempt to address the issues of unpredictable crises, rapid progression of thought to action, and method lethality, several public health-oriented programs have been developed that emphasize the importance of reducing high-risk individuals' access to lethal means. These prevention programs are referred to as lethal means reduction paradigms, and the implementation of these interventions have been shown to be effective at reducing death by suicide, with several international examples. According to Sinyor et al., ${ }^{28}$ restricting access to common methods of suicide may disrupt the process due to the fact that suicidal crises are often short-lived and people tend to report a preference for use of a specific method.

Until recently, self-poisoning by use of pesticides accounted for nearly $30 \%$ of suicides worldwide. ${ }^{29}$ In 1995 , Sri Lanka had considerably high rates of suicide, of which nearly two-thirds were due to the ingestion of pesticides. Toxic pesticides, like firearms, are highly lethal methods of suicide due to their high toxicity. Similar to easy access to firearms in the U.S., toxic pesticides were readily available for purchase in certain countries. Thus, in order to combat 
the increasing rates of suicide by pesticides, the World Health Organization (WHO) banned class I pesticides starting in $1984 .^{28}$ Data collected from the Department of Police, Division of Statistics, Sri Lanka in the year 2005, showed a nearly $50 \%$ decrease in suicide rates since the early 1990s, with no significant evidence suggesting the use of alternative methods. $^{30}$

Lubin et al. $^{31}$ reported that suicide rates in the Israeli Defense Force (IDF) were alarmingly high prior to testing means reduction interventions. Prior to the intervention, soldiers took their firearms on weekend leave. However, the IDF instituted a policy change in 2006, which required that military personnel leave their sidearms on base when they went home for the weekends. According to data compared from the years 2003-2005 and 2007-2008, suicide rates within the IDF decreased by as much as $40 \%$ on the weekends after the policy change, with no significant change in rates of suicide during weekdays when soldiers had typical access to their weapons. ${ }^{31}$

In 2017, Sinyor et al. $^{28}$ assessed the effectiveness of the suicide barrier that was placed on the Bloor Street Viaduct in Toronto, Canada, which is the second most frequented bridge for suicide-death worldwide, after the Golden Gate Bridge in San Francisco. Sinyor et al. ${ }^{28}$ studied the impact of this barrier after its initial completion by comparing records from the Office of the Chief Coroner of Ontario between January 1, 1993 and December 31, 2014. The barrier at the Bloor Street Viaduct was completed in 2003; data were classified accordingly such that the 11 years from January 1993 to December 2003 were considered before the implementation of the barrier and the 11 years from January 2004 to December 2014 were considered after the implementation of the barrier. ${ }^{28}$ Results indicated that per-capita suicide rates at the Bloor Street Viaduct decreased from 9.0 deaths per year prior to the barrier to 0.1 deaths per year after the barrier. ${ }^{28}$ These results demonstrate the significant impact that barriers have on death by suicide and suggest that the increase of potential barriers to suicide may reduce the number of overall attempts.

Additional actions that have been taken to reduce access to lethal means include the development of locked firearm storage units, firearm buy-back programs, restrictions on drug prescriptions, detoxification of gas, catalytic converter mandates on motor vehicles, and the construction of structural barriers to suicide. ${ }^{32}$ Thus, straightforward policy changes regarding access to lethal means impact suicide deaths and according to Sinyor et al., ${ }^{28}$ there are very few individuals who seek out alternative methods to die by suicide once their preferred method is no longer available. While there have been relatively few scaled up programs to reduce access to lethal means in the U.S., the Counseling on Access to Lethal Means training program is an exception. ${ }^{33}$

\section{Counseling on Access to Lethal Means and the benefits of gatekeeper training}

According to Sale et al., ${ }^{32}$ many mental health professionals have undergone generalized mental health training, but few have undergone training to specifically address suicide and lethal means reduction. In a study conducted by Betz et al. ${ }^{34}$ that sought to identify the frequency of lethal means assessments conducted at emergency departments (ED), findings indicated that out of roughly 800 patients with suicidal ideation, only $18 \%(n=145)$ had received an assessment pertaining to lethal means access. Furthermore, of the $18 \%$ who received a lethal means assessment, only $8 \%(n=11)$ had documentation that ED personnel discussed an action plan to reduce their access to lethal means. ${ }^{34}$ This suggests a critical need for professionals to receive increased training related to lethal means assessment, especially considering that EDs in the United States receive approximately 650,000 visits related to suicidal behavior per year. ${ }^{34}$ According to Betz et al., ${ }^{34}$ a better understanding of the role that lethal means assessments play in risk assessments for suicidal patients may help to reduce death by suicide. Consequently, the Counseling on Access to Lethal Means (CALM) training program was developed.

The purpose of the CALM program is to train mental health professionals on the importance of reducing access to lethal means among those at risk for suicide. ${ }^{33}$ The program created by Elaine Frank and Mark Ciocca appropriately targets reducing access to firearms, given they are the leading method of suicide and the method with the highest rates of fatality in the United States. ${ }^{20}$ The clinical training program lasts approximately two to three hours and teaches clinicians about how reducing access to lethal means can prevent suicide. In addition, the program focuses on training mental health care providers on how to effectively communicate with at-risk clients and their family members on the importance of temporarily reducing access to lethal means during a crisis. Clinicians are shown video re-enactments of lethal means assessments and are encouraged to role-play with their colleagues so that they may practice these skills. Additionally, clinicians are informed of the prevalence of suicide, different types of lethal means, examples of effective lethal means reduction efforts, and a detailed instruction on how to most effectively work with clients who are at imminent risk of harming themselves and also possess firearms or other lethal means. ${ }^{32}$ Overall, after an initial trial of the program, 65\% of clinicians reported having used CALM techniques six months after the training. Moreover, they reported greater knowledge and confidence regarding counseling clients about reducing access. ${ }^{33}$

Another study conducted by Sale et al. $^{32}$ found that out of 399 mental health professionals who attended a CALM training, $54 \%$ of participants reported having discussed lethal means reduction with suicidal clients at baseline, while $74 \%$ reported having discussed means reduction with suicidal clients after having attended the CALM training. Additionally, there was a main effect of time, indicating that participants showed significant gains in comfort, knowledge, and intent to discuss lethal means with their clients after the training. ${ }^{32}$ These findings show that as clinicians become more comfortable talking about lethal means reduction with their clients, they are more likely to ask direct questions related to means reduction when clients are in crises. ${ }^{32}$ 
While the results of CALM clinical trainings have shown promise, these results have not been replicated broadly enough and there is no empirical evidence available regarding a gatekeeper version of CALM.

Gatekeeper training is a key component of universal suicide prevention that consists of educating non-mental health professionals such as police officers, paramedics, first responders, teachers, coworkers, college resident assistants and peers on the basics of suicide prevention. The assumption behind gatekeeper training is that non-mental health professionals and paraprofessionals are often among the first to come in contact with high-risk individuals and can intervene when clinicians are not present or available. Gatekeeper training is typically less intense than clinical training in that sessions are shorter and put fewer demands on the trainees. Nevertheless, various gatekeeper programs have demonstrated improvements in knowledge, attitudes, and selfperceived confidence regarding suicide prevention skills. For example, in a study conducted in the United Kingdom, a gatekeeper training for police officers was evaluated to determine its impact on trainee confidence in conducting suicide prevention interventions. ${ }^{35}$ The program consisted of a fourhour session, a training manual, and a PowerPoint presentation including case vignettes and reflective questions to encourage group discussions and learning. Questionnaires evaluating knowledge and confidence regarding suicide prevention were administered before and after the training, which revealed statistically significant increases in both components. Although gatekeeper trainings have long been a part of suicide prevention programs, most focus on improving knowledge and attitudes regarding suicide prevention overall, rather than on lethal means reduction specifically.

\section{The present study}

Given this gap in the literature, the primary aim of the present study was to evaluate whether a gatekeeper version of the CALM training would have an effect on RAs' confidence in delivering suicide prevention and means reduction interventions among college students. We specifically chose to administer this training to RAs, as the rate of suicide among adolescents, young adults, and college students has increased significantly in the past several years. ${ }^{23}$ The training was evaluated via self-reported knowledge and confidence levels regarding: 1) conducting suicide prevention overall; and 2) conducting means reduction-based interventions, specifically. We modeled the methodology used in the Marzano et al. ${ }^{35}$ study and utilized four suicide prevention items from their questionnaire and added an additional follow-up interval as part of the investigation. We used three items from the original Johnson et al. ${ }^{33}$ CALM questionnaire regarding confidence in conducting means reduction counseling as an attempt to replicate the findings in our study. We also measured confidence in suicide prevention and means reduction interventions at three different time points (pre, post, follow-up) as compared to the Johnson et al. ${ }^{33}$ study, which included pre-and post-training questions in the same survey at the conclusion of the training.
Given the empirical evidence that suggests lethal means reduction interventions are effective internationally (e.g., Sri Lanka, IDF, Bloor Street Viaduct) as well as the success of various gatekeeper programs and a clinical means reduction program in the U.S., we assessed the training effects of a gatekeeper version of the CALM program. We hypothesized that in accordance with other lethal means and gatekeepertraining studies cited above, our program would be associated with increased confidence in conducting suicide prevention and means reduction interventions after the training when compared to baseline levels of confidence among a sample of university RAs.

\section{Method}

\section{Participants}

The participants were 167 RAs from a university located in the Southeastern United States. Participants attended a gatekeeper version of the CALM training and learned skills regarding suicide prevention and means reduction. RAs are undergraduate students who are responsible for maintaining order and safety within on-campus dormitories, in addition to providing various resources for their residents. Among the 167 participants who attended the training, 141 (48 males and 93 females) consented to participate in the current study and all participants were over the age of 18 $(M=20.24, S D=1.07)$. No compensation was offered for participation and while RAs were required to attend training for their orientation, participants had the opportunity to opt out of involvement in the current study via a fully informed consent procedure that was completed prior to data collection. The university's Institutional Review Board (IRB) determined this study to be exempt from IRB oversight on December 12, 2016 (Study number 17-0167).

\section{Measures}

Participants responded to a modified version of the Confidence in Suicide Prevention Measure, which was developed by Marzano, the lead psychologist behind the Police and Suicide Prevention study conducted in the United Kingdom. ${ }^{35}$ We obtained permission from Marzano to use her instrument and revised the measure to include three items regarding means reduction counseling from the Johnson et al. ${ }^{33}$ study. The updated measure accounted for demographic information by requesting age, gender, number of years of experience as an RA, and college major. Furthermore, the measure accounted for previous involvement in suicide prevention and other mental health training by asking about relevant experience. Following the demographics portion, confidence and knowledge in suicide prevention and means reduction counseling were evaluated via a 5-point continuous Likert Scale ( $5=$ "strongly agree," $1=$ "strongly disagree"; with higher scores suggestive of greater confidence). 


\begin{tabular}{|c|c|c|c|}
\hline Items & Baseline & Post-training & Follow-up \\
\hline \multicolumn{4}{|l|}{ Suicide prevention composite } \\
\hline "I feel I can accurately identify situations where a person is at risk of suicide." & $4.04(0.559)$ & $4.33(0.504)$ & $4.24(0.758)$ \\
\hline "I know how to approach and question people at risk of suicide." & $3.83(0.870)$ & $4.29(0.636)$ & $4.15(0.870)$ \\
\hline "I feel comfortable assessing someone for suicide risk." & $3.64(0.951)$ & $4.15(0.786)$ & $3.97(0.964)$ \\
\hline "I know how to refer people at risk of suicide to the services most appropriate to their needs and level of risk." & $4.16(0.816)$ & $4.44(0.570)$ & $4.39(0.812)$ \\
\hline \multicolumn{4}{|l|}{ Means reduction composite } \\
\hline "I am familiar with means reduction approaches to suicide prevention." & $3.30(0.985)$ & $4.37(0.545)$ & $4.14(0.899)$ \\
\hline "Suicide can be prevented by reducing access to lethal means." & $3.25(0.990)$ & $4.27(0.814)$ & $4.01(1.056)$ \\
\hline "I am confident in my ability to talk to people about reducing access to lethal means." & $3.38(0.930)$ & $4.17(0.712)$ & $4.00(0.871)$ \\
\hline
\end{tabular}

Note: All items were presented on a Likert Scale with higher scores suggestive of greater confidence ( $5=$ Strongly Agree, $1=$ Strongly Disagree).

\section{Procedure}

After informed consent was acquired, participants completed the Suicide Prevention Training: Learning \& Development Evaluation Form at baseline. In order to adapt the original CALM training, the authors developed a shorter version relevant to college students. The gatekeeper training lasted approximately one hour and consisted of a PowerPoint presentation wherein the trainers (KDM, JPJ) discussed the principles of CALM and the importance of reducing access to lethal means. Immediately after the training, the questionnaire was administered a second time.

All consenting individuals received a follow-up evaluation via Qualtrics approximately 4-6 weeks after the initial training in order to measure changes in knowledge and confidence levels regarding suicide prevention (SP) and means reduction $(\mathrm{MR})$ interventions.

\section{Primary analyses}

All analyses were performed using IBM SPSS statistics version $24 .^{36}$ We ran basic descriptive and frequency analyses regarding the demographics of our sample. We also assessed internal reliability (Chronbach's alpha) for SP and MR items separately. After computing composite SP (4 items) and MR (3 items) scores, a repeated-measures ANOVA was conducted to determine whether there was a main effect for time. Following that, six paired samples post-hoc t-tests were conducted to assess the effects between the various intervals for the two constructs (baseline vs. post-training, post-training vs. follow-up, baseline vs. follow-up). The critical $p$ value was .008 after adjusting for Bonferroni corrections. Using these results, Cohen's $d$ effect sizes were computed (95\% confidence intervals), utilizing Cohen's ${ }^{37}$ suggested interpretive guidelines for effect sizes $(.2=$ small; $.5=$ medium; $.8=$ large) .

\section{Results}

Means and standard deviations for questionnaire items are presented in Table 1. The number of participants varied at each time point, such that: baseline $(n=141)$, post-training $(n=131)$, and follow-up $(n=88)$. Overall, there was a $92.9 \%$ response rate from baseline to post-training, and a $66.4 \%$ response rate from post-training to follow-up.

In order to assess the internal consistency for each construct we conducted a reliability analysis for SP and MR constructs at each time point. The SP item internal consistency coefficients were moderate to high: $(\alpha=.822$, baseline; $\alpha=.850$, post-training; $\alpha=.918$, follow-up), whereas the MR item internal consistency coefficients were somewhat lower to moderate: $(\alpha=.662$, baseline; $\alpha=.673$, posttraining; $\alpha=.828$, follow-up).

We computed overall mean composites for our two constructs and found a similar pattern for SP and MR (see Figure 1; wherein higher scores are suggestive of higher levels of confidence). On average, RAs felt moderately confident in suicide prevention skills at baseline $(M=15.77$, $S D=2.39$ ), and their confidence increased at post-training $(M=17.27, S D=1.90)$, while confidence levels evidenced a slight decay after a 4-6week follow-up $(M=16.62$, $S D=3.10)$. Similar to SP confidence levels, RAs felt relatively confident regarding $\mathrm{MR}$ interventions at baseline $(M=9.65, \quad S D=2.16)$, and improved post-training $(M=12.90, S D=1.60)$, while confidence levels once again displayed a slight decay after a 4-6week follow-up $(M=12.10, S D=2.51)$. These patterns illustrate the phenomenon that confidence levels increased at post-training and revealed a slight regression toward baseline approximately 4-6 weeks after the training. However, the amount of decay did not return to baseline levels of confidence.

Repeated measures ANOVAs (with Greenhouse-Geisser corrections) were completed for the composites and in both instances, there was a statistically significant main effect for time: SP, $F(1.448,115.814)=14.047, p=.001, \eta^{2}=.149$; $\mathrm{MR}, F(1.592,128.924)=86.527, p=.001, \eta^{2}=.516$. Posthoc paired samples t-tests and Cohen's $d$ effect sizes were computed for comparisons between intervals (Bonferroni correction: $p=.008$; see Table 2 ). The SP baseline to posttraining comparison was statistically significant $(p<.001)$ with a medium effect $(d=.654,95 \% \mathrm{CI}=.406-.903)$; the MR baseline to post-training was also statistically significant $(p<.001)$ resulting in a large effect $(d=1.503,95 \% \mathrm{CI}=$ 1.227 - 1.78). In contrast, the SP post-training to follow-up revealed a non-statistically significant and small decay in the training effect $(p=.037, d=-.254,95 \% \mathrm{CI}=-.564-$ $.055)$, whereas the MR post-training to follow-up comparison showed a statistically significant decay $(p=.001)$ but small effect $(d=-.383,95 \% \mathrm{CI}=-.692--.074)$. The SP baseline to follow-up comparison was statistically significant $(p=.008)$ but the effect size was small $(d=.323,95 \%$ $\mathrm{CI}=.022-.624)$, whereas the MR baseline to follow-up comparison $(p<.001)$ was large $(d=1.035,95 \%$ CI $=.721$ $-1.35)$. 


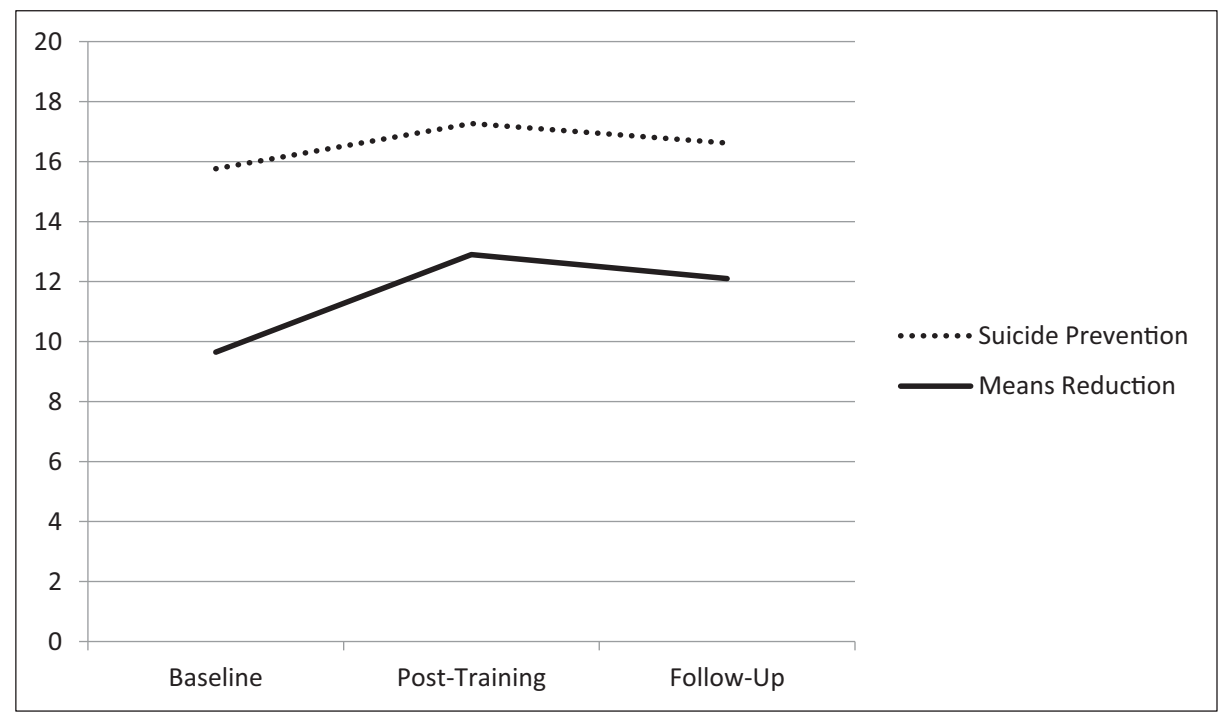

Figure 1. Composite means for change in confidence over time (higher scores reflect more confidence).

Table 2. Post-hoc paired samples t-tests.

\begin{tabular}{|c|c|c|c|c|c|}
\hline \multirow[t]{2}{*}{ Composite } & \multicolumn{2}{|c|}{ Interval means $(S D)$} & \multirow[t]{2}{*}{$p^{*}$} & \multirow[t]{2}{*}{ Cohen's $d^{* *}$} & \multirow[t]{2}{*}{$95 \% \mathrm{Cl}$} \\
\hline & Baseline & Post-training & & & \\
\hline Means reduction & $9.85(2.25)$ & $12.81(1.64)$ & $.000^{*}$ & 1.503 & $1.227-1.78$ \\
\hline \multirow[t]{2}{*}{ Means reduction } & $12.90(1.60)$ & $12.10(2.51)$ & $.001 *$ & -0.383 & $-0.692--0.074$ \\
\hline & Baseline & Follow-Up & & & \\
\hline Suicide prevention & $15.81(2.36)$ & $16.70(3.06)$ & $.008^{*}$ & 0.323 & $0.022-0.624$ \\
\hline
\end{tabular}

Note: $\mathrm{SD}=$ Standard Deviation.

*Significant levels based on Bonferroni corrections $(p=.008)$.

$* *$ Small effect size (.2), medium effect size (.5), large effect size (.8).

\section{Discussion}

The results were suggestive of modest to large training effects for suicide prevention and means reduction confidence levels among a sample of college RAs following a brief, gatekeeper CALM training. The improvements in confidence were larger for the means reduction items, which might be due to the fact that participants were learning new material for the first time. That is, compared to typical suicide prevention concepts, they were less familiar with means reduction interventions and therefore had more room for growth. Although lethal means reduction programs are certainly not new to some groups such as public health professionals who have frequently adopted community or population-based interventions with success, gatekeepers and clinicians accustomed to individual interventions do not typically approach suicide prevention in such a systemic manner. ${ }^{38}$ Therefore, it would make sense that confidence levels increased following a training session during which individuals learned novel material. Basic suicide prevention education is typically required for RA training, which is indicative of the possibility that this population has had some prior experience dealing with suicide prevention techniques. This might explain why confidence levels were relatively high during baseline.

A similar and consistent pattern was evident for both suicide prevention and means reduction composites, suggesting that RAs felt more confident immediately after the CALM training. Nonetheless, there was a small decay of the training effects at follow-up. These results could be attributable to the mere passage of time, but confidence appraisals did not return to baseline levels. Essentially, confidence levels increased directly after the CALM training and decreased slightly over time. Yet overall, there was an increase in confidence for both SP and MR from baseline to follow-up and the larger effects for MR were observed just like they were between baseline and post-training. These data directly reflect findings from the Sale et $\mathrm{al}^{32}$ study that found that clinicians' confidence in discussing means reduction with their clients increased directly after their CALM training, but significantly decreased after a follow-up period. These findings might suggest that trainings and booster sessions should be provided on a more consistent basis to help sustain the impact and to maintain confidence and knowledge.

In an attempt to address this deterioration of confidence and knowledge among participants who attend gatekeepertraining programs, Shtivelband, Aloise-Young, and Chen ${ }^{39}$ conducted a study that sought to identify methods to improve retention of information. In-depth interviews and focus groups were conducted among a sample of 44 gatekeepers and data were analyzed qualitatively. Results indicated that post-training interventions may prove to be more effective if they include the following themes: "(1) social 
network - connecting with other gatekeepers; (2) continued learning - further education; (3) community outreach building awareness; (4) accessibility - convenience; (5) reminders - ongoing communication; (6) program improvement - enhancing previous training; and (7) certification accreditation." ${ }^{39}$

There were some notable limitations in the current study. For example, the sample was restricted to RAs and it is unclear how these results might be generalized to other gatekeeper groups (e.g., firefighters, emergency personnel). Another notable limitation of the current study was the lack of a control group. Future studies should attempt to establish a control condition so that researchers can confidently and definitively demonstrate that the CALM training is the sole influencer of subsequent results. In addition, although there was an effect on RA confidence levels, we had no way of determining whether the training had an effect on real behavior in practice. Future research should include larger, more diverse samples and attempts to replicate the training effect over longer intervals. Furthermore, empirical inquiries should be conducted to determine the association between gatekeeper trainings on future suicide prevention behaviors among those who attend the CALM trainings. If CALM becomes implemented more broadly, rates of suicide should be studied in order to determine the impact of lethal means reduction approaches for suicide prevention.

According to Sale et al., ${ }^{32}$ the CALM training is currently listed on the Suicide Prevention Resource Center (SPRC) Best Practices Registry (BPR) under Adherence to Standards but is not currently considered an "evidence-based" practice due to the limited number of studies that have been conducted regarding its effectiveness. Therefore, it is imperative for future researchers who study suicide prevention initiatives to focus their efforts on unveiling the potential strengths and limitations of both the gatekeeper and clinical versions of this training. A greater number of empirical studies that evaluate CALM may lead to a greater number of clinicians and gatekeepers implementing means reduction techniques in their practice and real-life behavior, and thus fewer deaths by suicide.

Overall, the findings from the current study supported our hypothesis that the CALM gatekeeper training would be associated with higher confidence levels regarding suicide prevention and means reduction interventions among a sample of college RAs. Moreover, these data mirror some of the training effects from previous lethal means reduction studies. ${ }^{32}$ It is vital to continue studying the benefits of lethal means reduction programs given its documented history of preventing death by suicide. It is also imperative that suicide prevention efforts continue to target the increasingly at-risk population of college students. While it is unlikely that any prevention program or intervention will completely eradicate suicide, there is strong evidence to suggest that means reduction interventions can prevent devastating consequences and create better opportunities for intervention. To this end, broadening the exposure of college health and residence life staff to potentially life-saving training is equally important, especially if the benefits of trainings like
CALM that have been shown to increase confidence, also impact actual behavior. While additional research is still needed, the implications of the current study suggest that the CALM gatekeeper program had a positive effect on suicide prevention confidence levels among a sample of RAs.

\section{Conflict of interest disclosure}

The authors have no conflicts of interest to report. The authors confirm that the research presented in this article met the ethical guidelines, including adherence to the legal requirements, of the United States and received approval from the Institutional Review Board of Appalachian State University.

\section{References}

1. Centers for Disease Control and Prevention. National vital statistics report. https://www.cdc.gov/nchs/data/nvsr/nvsr68/nvsr68_ 09-508.pdf. Published June 24, 2019. Accessed February 12, 2020.

2. American Foundation for Suicide Prevention. Suicide statistics. https://afsp.org/about-suicide/suicide-statistics/. Published 2017. Accessed December 15, 2018.

3. Eisenberg D, Lipson SK, Beck K, Dalal M, Despot O. The healthy minds study: [dataset]. Healthy Minds Network/ Research/Data for Researchers/Database. http://healthymindsnetwork.org/system/resources/ W1siZiIsIjIwMTcvMDcvMjgvMDlfNTJfMDNfNDMwX0hNU19uYXRpb25hbF9EYXRhUmVwb3J0XzIwMTQucGRmIl1d/HMS_ national_DataReport_2014.pdf. Published 2014. Accessed February 28, 2019.

4. Mortier P, Cuijpers P, Kiekens G, et al. The prevalence of suicidal thoughts and behaviours among college students: a metaanalysis. Psychol Med. 2018;48(4):554-565. doi:10.1017/ S0033291717002215.

5. Dueweke AR, Schwartz-Mette RA. Social-cognitive and socialbehavioral correlates of suicide risk in college students: contributions from interpersonal theories of suicide and depression. Arch Suicide Res. 2018;22(2):224-240. doi:10.1080/13811118.2017. 1319310.

6. De Luca SM, Franklin C, Yueqi Y, Johnson S, Brownson C. The relationship between suicide ideation, behavioral health, and college academic performance. Community Ment Health J. 2016; 52(5):534-540. doi:10.1007/s10597-016-9987-4.

7. Lockman JD, Servaty-Seib HL. College student suicidal ideation: perceived burdensomeness, thwarted belongingness, and meaning made of stress. Death Stud.. 2016;40(3):154-164. doi:10.1080/ 07481187.2015.1.105325.

8. Ploskonka RA, Servaty-Seib HL. Belongingness and suicidal ideation in college students. J Am Coll Health. 2015;63(2):81-87. doi: 10.1080/07448481.2014.983928.

9. Stewart JG, Shields GS, Esposito EC, Cosby EA, et al. Life stress and suicide in adolescents. J Abnorm Child Psychol. 2019;47(10): 1707-1722. doi:10.1007/s10802-019-00534-5.

10. Letarte CM. Keepers of the night: the dangerously important role of resident assitants on college and university campuses. Kentucky J Higher Educ Policy Pract. 2013;2(4):4.

11. Shannonhouse L, Dennis Lin Y, Shaw K, Wanna R, Porter M. Suicide intervention training for college staff: program evaluation and intervention skill measurement. J Am Coll Health. 2017; 65(7):450-456. doi:10.1080/07448481.2017.1341893.

12. Tompkins TL, Witt J. The short-term effectiveness of a suicide prevention gatekeeper training program in a college setting with residence life advisers. J Primary Prevent. 2009;30(2):131-149. doi:10.1007/s10935-009-0171-2.

13. QPR Institute. Question. Persuade. Refer. https://www.qprinstitute.com. Published 2017. Accessed December 27, 2018. 
14. Borges G, Angst J, Nock MK, Ruscio AM, Walters EE, Kessler RC. Risk factors for twelve-month suicide attempts in the national comorbidity survey replication (NCS-R). Psychol Med. 2006;36(12):1747-1757. doi:10.1017/S0033291706008786.

15. Miller M, Barber C, White RA, Azrael D. Firearms and suicide in the united states: is risk independent of underlying suicidal behavior? Am J Epidemiol. 2013;178(6):946-955. doi:10.1093/aje/ kwt197.

16. Werbeloff N, Dohrenwend BP, Levav I, et al. Demographic, behavioral, and psychiatric risk factors for suicide: a 25 -year longitudinal cohort study. Crisis J Crisis Intervention Suicide Prev. 2016;37(2):104-111. doi:10.1027/0227-5910/a000359.

17. Swanbrow Becker MA, Blankenship P, Melo K, et al. Improving resident assistant suicide prevention gatekeeper training through focus group feedback. Coll Stud Aff J. 2017;35(2):117-130. doi: 10.1353/csj.2017.0017.

18. Deisenhammer EA, Ing C, Strauss R, Kemmler G, Hinterhuber $\mathrm{H}$, Weiss EM. The duration of the suicidal process: how much time is left for intervention between consideration and accomplishment of a suicide attempt? J Clin Psychiatry. 2009;70(1): 19-24. doi:10.4088/JCP.07m03904.

19. Simon TR, Swann AC, Powell KE, Potter LB, Kresnow M-j, O'Carroll PW. Characteristics of impulsive suicide attempts and attempters. Suicide Life Threatening Behav. 2001;32:49-59. doi: 10.1521/suli.32.1.5.49.24212.

20. Sorenson SB, Vittes KA. Mental health and firearms in community-based surveys: implications for suicide prevention. Eval Rev. 2008;32(3):239-256. doi:10.1177/0193841X09315871.

21. Kattimani S, Sarkar S, Menon V, Muthuramalingam A, Nancy P. Duration of suicide process among suicide attempters and characteristics of those providing window of opportunity for intervention. J Neurosci Rural Pract. 2016;7(04):566-570. doi:10.4103/ 0976-3147.185505.

22. Anestis MD, Capron DW. Deadly experience: the association between firing a gun and various aspects of suicide risk. Suicide Life Threat Behav. 2017;48(6):699-708. doi:10.1111/sltb.12381.

23. Lee LK, Mannix R. Increasing fatality rates from preventable deaths in teenagers and young adults. JAMA. 2018;320(6):543. doi:10.1001/jama.2018.6566.

24. Centers for Disease Control and Prevention. CDC WONDER. https://wonder.cdc.gov. Published December 18, 2018. Accessed December 27, 2018.

25. United States Census Bureau. Census Bureau Projects U.S. Population of 312.8 Million on New Years Day. https://www.census.gov/newsroom/releases/archives/population/cb11-219.html. Published December 19, 2011. Accessed December 27, 2018.

26. May AM, Klonsky ED. "Impulsive" suicide attempts: what do we really mean? Pers Disord Theory Res Treat. 2016;7(3):293-302. doi:10.1037/per0000160.
27. Betz ME, Barber C, Miller M. Suicidal behavior and firearm access: results from the second injury control and risk survey. Suicide Life Threatening Behav. 2011;41(4):384-391. doi:10.1111/ j.1943-278X.2011.00036.x.

28. Sinyor M, Schaffer A, Redelmeier DA, et al. Did the suicide barrier work after all? Revisiting the bloor viaduct natural experiment and its impact on suicide rates in Toronto. BMJ Open. 2017;7(5):e015299. doi:10.1136/bmjopen-2016-015299.

29. Gunnel D, Eddleston M, Phillips MR, Konradsen F. The global distribution of fatal pesticide self-poisoning: systematic review. BMC Public Health. 2007;7(1):1-15. doi:10.1186/1471-2458-7357.

30. Knipe DW, Metcalfe C, Fernando R, et al. Suicide in Sri Lanka 1975-2012: age, period and cohort analysis of police and hospital data. BMC Public Health. 2014;14(1):1-13. doi:10.1186/14712458-14-839.

31. Lubin G, Werbeloff N, Halperin D, Shmushkevitch M, Weiser $\mathrm{M}$, Knobler H. Decrease in suicide rates after a change of policy reducing access to firearms in adolescents: a naturalistic epidemiological study. Suicide Life Threatening Behav. 2010;40(5): 421-424. doi:10.1521/suli.2010.40.5.421.

32. Sale E, Hendricks M, Weil V, Miller C, Perkins S, McCudden S. Counseling on access to lethal means (CALM): an evaluation of a suicide prevention means restriction training program for mental health providers. Community Ment Health J. 2017;54(3): 293-301. doi:10.100y/s10597-017-0190-z.

33. Johnson RM, Frank EM, Ciocca M, Barber CW. Training mental healthcare providers to reduce at-risk patients' access to lethal means of suicide: evaluation of the CALM project. Arch Suicide Res. 2011;15(3):259-264. doi:10.1080/13811118.2011.589727.

34. Betz ME, Kautzman M, Segal DL, et al. Frequency of lethal means assessment among emergency department patients with a positive suicide risk screen. Psychiatry Res. 2018;260:30-35. doi: 10.1016/j.psychres.2017.11.038.

35. Marzano L, Smith M, Long M, Kisby C, Hawton K. Police and suicide prevention: evaluation of a training program. Crisis. 2016;37(3):194-204. doi:10.1027/0227-5910/a000381.

36. IBM Corp [computer software] Version 24.0. Armonk, NY: IBM; 2016.

37. Cohen J. Statistical Power Analyses for the Behavioral Sciences. 2nd ed. Hillsdale, NJ: Lawrence Earlbaum Associates; 1988.

38. Yip PSF, Caine E, Yousuf S, Chang S, Wu KC, Chen Y. Means restriction for suicide prevention. Lancet. 2012;379(9834): 2393-2399. doi:10.1016/S0140-6736(12)60521-2.

39. Shtivelband A, Aloise-Young PA, Chen PY. Sustaining the effects of gatekeeper suicide prevention training. Res Trends. 2015;36(2): 102-109. doi:10.1027/0227-5910/a000304. 